\title{
Modified double lumen tube for a unique bronchial and carinal resection in a patient undergoing uniportal VATS for tumour: A case report
}

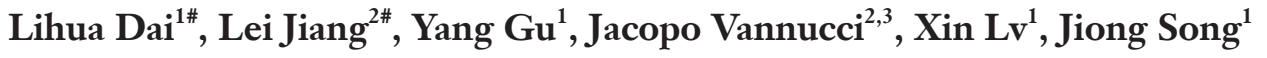 \\ ${ }^{1}$ Department of Anesthesiology, ${ }^{2}$ Department of Thoracic Surgery, Shanghai Pulmonary Hospital, Tongji University, Shanghai 200433, China; \\ ${ }^{3}$ Department of Thoracic Surgery, University of Rome Sapienza, Policlinico Umberto I, Rome, Italy \\ \#These authors contributed equally to this work. \\ Correspondence to: Jiong Song. Department of Anesthesiology, Shanghai Pulmonary Hospital, No. 507 Zhengmin Rd., Yangpu District, Shanghai \\ 200433, China. Email: sw480@126.com.
}

\begin{abstract}
For bronchial sleeve and carinal resection and reconstruction during uniportal video-assisted thoracic surgery (VATS), ventilation technique remains a demanding challenge for the anesthesiologists. The ventilation techniques require maintaining adequate gas exchange while providing a good surgical exposure. The case we present was a 58-year-old female with carcinoma in the right upper lobe involving the right main bronchus and the lower trachea. Right upper sleeve lobectomy, carinal resection and reconstruction was performed under uniportal VATS. A modified double-lumen tube (DLT) was inserted to achieve one-lung ventilation, and high-frequency jet ventilation (HFJV) was passed through the DLT to provide oxygenation during the anastomosis without interfering with the surgical procedure. The whole procedure was uneventful. We suggest that the double lumen tube could be modified being a simple and safe option for one-lung ventilation in carinal resection and reconstruction under uniportal VATS.
\end{abstract}

Keywords: Case report; carinal resection; double-lumen endotracheal tube; uniportal video-assisted thoracic surgery (VATS)

Submitted Dec 13, 2019. Accepted for publication Dec 30, 2019.

doi: $10.21037 /$ tcr.2019.12.90

View this article at: http://dx.doi.org/10.21037/tcr.2019.12.90

\section{Introduction}

Uniportal video-assisted thoracic surgery (VATS) is a minimally invasive procedure that has many potential advantages, including less postoperative pain, faster recovery and a better cosmetic incision (1). The thoracic surgeries by uniportal VATS approach necessitate lung isolation and one-lung ventilation (OLV) to enhance the surgical view and provide adequate operating space. Thoracoscopic surgeries involving the carina pose more challenges to the anesthesiologists, as it requires precise airway management to maintain sufficient airflow in the distal airways.

Cross-field intubation was traditionally used in the carinal resection and reconstruction surgeries (2). But it has the defect of interference of the surgical field under uniportal VATS and needs an additional incision.
One-lung ventilation in the thoracic surgical patient can be achieved with the use of a double-lumen endotracheal tube (DLT). In carinal surgeries, its wide external diameter and the long tracheal cuff would bring excessive tension during the reconstruction anastomosis. For this we describe the successful use of a modified DLT for one-lung ventilation for the right upper lobectomy combined with carinal resection and reconstruction under uniportal VATS. We present the following case in accordance with the CARE Guideline.

\section{Case presentation}

A 58 -year-old female (161 cm in height, $65 \mathrm{~kg}$ in weight) was referred to our service for cough with bloody sputum. 

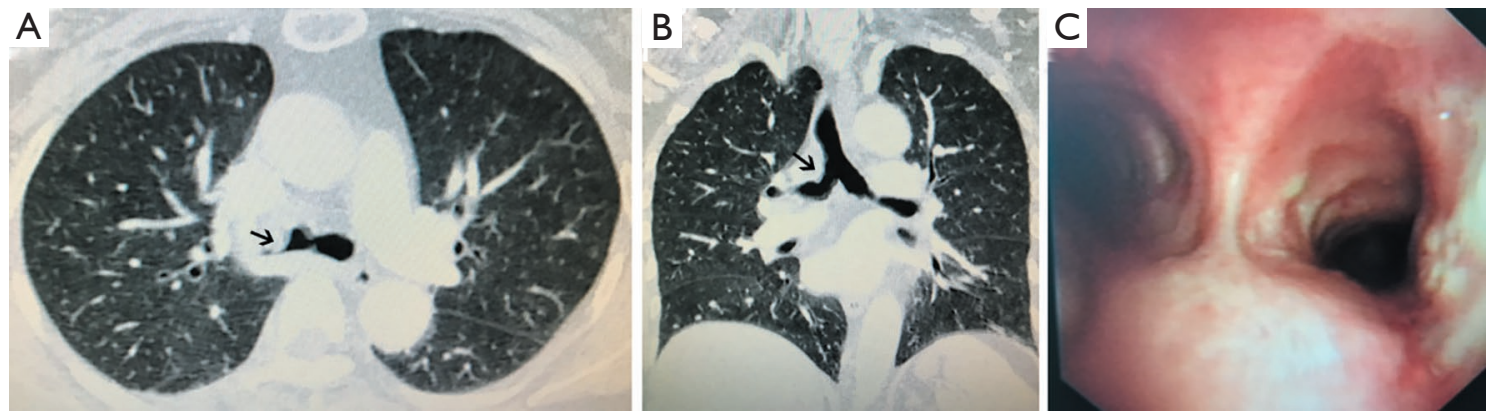

Figure 1 Imaging features of the patient. (A) Computed tomography shows a soft tissue shadow and local thickening wall of the lower trachea and the right main bronchus. The tumour was pointed out with black arrows; (B) coronal scan of the tumour location. The tumour was pointed out with black arrows; (C) bronchoscopic view of the involved lateral wall of the distal trachea and right main bronchus.

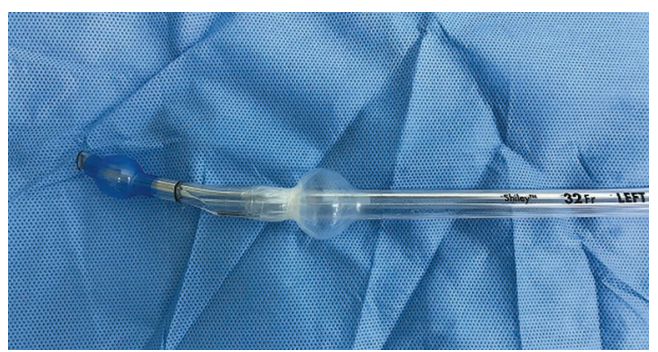

Figure 2 The modified 32 Fr left double-lumen tube. The distal end of the tracheal cuff was wrapped with the $3 \mathrm{M}$ Tegaderm transparent film.

The chest computed tomography scan showed a soft tissue shadow and thickened wall in lower part of the trachea and the right main bronchus (Figure 1A,B). The bronchoscopy revealed a tumour encroaching into the terminal trachea and right main bronchus (Figure 1C) that has been a biopsy proven mucoepidermoid carcinoma. To better preserve the pulmonary function, right upper sleeve lobectomy and carinal resection and reconstruction under uniportal VATS was planned for this patient.

The patient had no reported comorbidities and was rated physical classification II according to the American Society of Anesthesiologists.

A left-sided double-lumen tube (DLT) size $32 \mathrm{Fr}$ was prepared and modified in advance, with its distal end of the tracheal cuff wrapped with some transparent $3 \mathrm{M}$ Tegaderm ${ }^{\mathrm{TM}}$ film in order to reduce the vertical length of the cuff thus preventing tumour/tube contact and allowing for endotracheal maneuvers like suctioning (Figure 2). Hence, this modified tube could help the procedure by providing a triple function including one-lung ventilation, synergy between tumour and ventilation asset, and suctioning below the tracheal cuff. In case of malfunction or need, an extra-long small bore endotracheal tube (ID $5.0 \mathrm{~mm}$ ) was ready for cross-filed intubation.

After anesthesia induction, the modified DLT mentioned above was lubricated and selectively intubated to the left main bronchus (LMB). The tube position was confirmed by both auscultation and fiberoptic bronchoscopy, and the patient was moved to a lateral decubitus position.

The chest was entered through the fourth intercostal space. After ligation and cutting off the right superior pulmonary vein and artery branch to the upper lobe, the right upper lung was resected en bloc the involved right main bronchus and right lateral wall of the lower trachea. And the DLT was partially exposed to the surgical field and its position could be adjusted with a thoracoscopic forcep by the operator (Figure 3). Then the lower part of the trachea and the left main bronchus was dissected circumferentially by scalpel and scissors. After frozen section analysis of margins, the carina reconstruction was initiated. The DLT was pulled out of the surgical field to the trachea. A suction catheter hosting to the high-frequency jet ventilator was inserted through the DLT into the left main bronchus. The jet ventilator delivered $100 \% \mathrm{O}_{2}$ with a respiratory rate of 110 times per minute, a driving pressure of $0.3 \mathrm{MPa}$ and I:E ratio of 1:1. The distal trachea and left main bronchus were anastomosed under high-frequency jet ventilation (HFJV) ventilation in an end-to-end fashion with continuous 3-0 prolene running sutures, starting from the membranous portion to the left side of trachea wall. When two thirds of the circumference were anastomosed, remaining an oval opening mirroring the bronchus intermedius lumen, the HFJV catheter was removed and the DLT was carefully advanced across the anastomosis line to the left main bronchus to carry out the conventional 

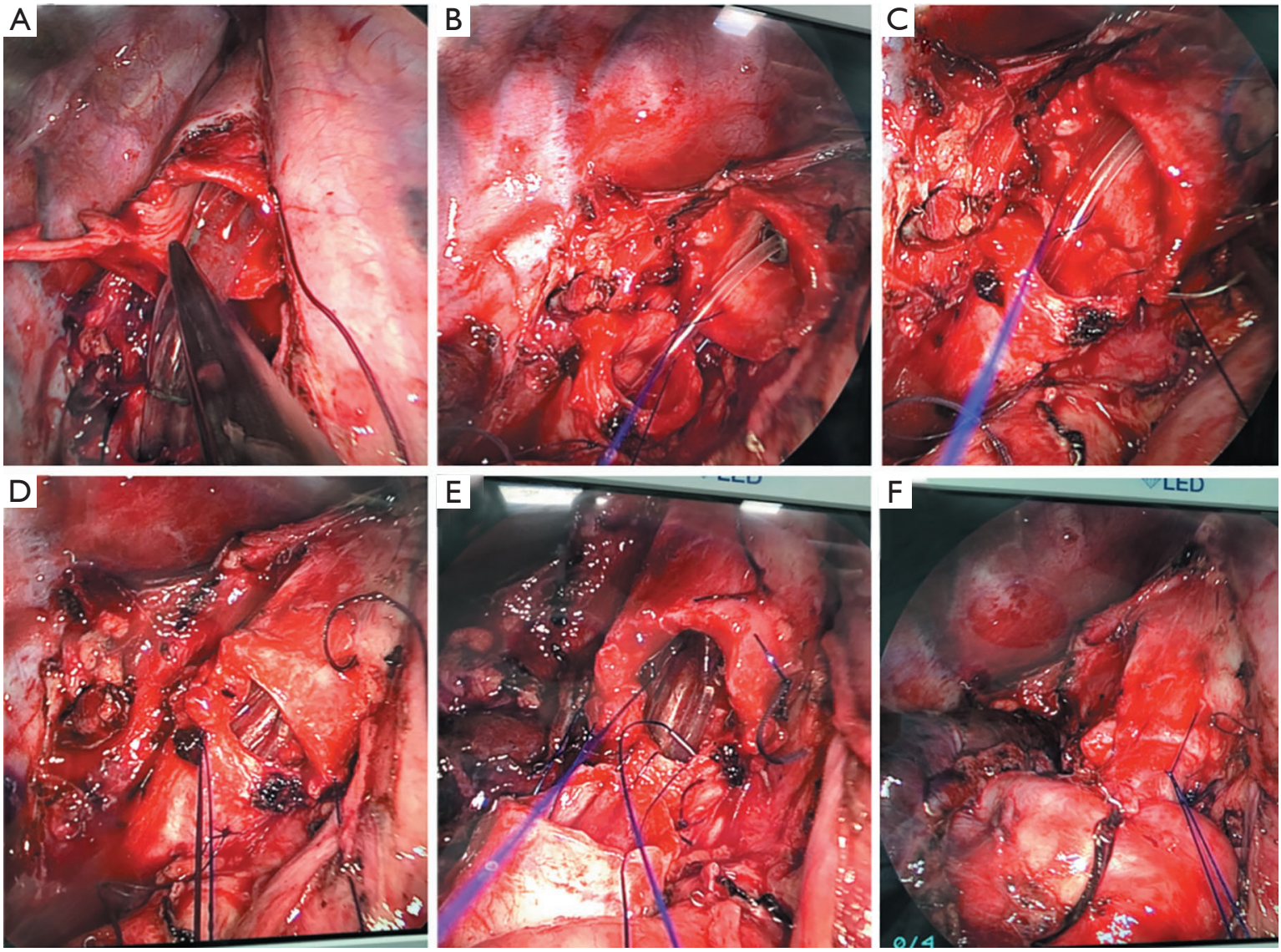

Figure 3 Ventilation technique during carinal resection and reconstruction. (A) DLT was initially inserted in the left-side and was partially exposed to the surgical field after removing the right upper lobe and carina; (B) DLT was pulled back to the trachea, instead, a highfrequency jet ventilation catheter was inserted through the DLT to the left main bronchus; (C) the anastomosis of the LMB to the trachea under high-frequency jet ventilation (HFJV); (D) DLT was reinserted to restart one-lung ventilation after the completion of trachea-LMB anastomosis; (E) view of the trachea, LMB, bronchus intermedius and accomplishment of the neo-carina; (F) final neo-carina. DLT, doublelumen endotracheal tube; LMB, left main bronchus.

one-lung ventilation. Subsequently the right bronchus intermedius was anastomosed to the opening in an end-to side fashion with continuous 3-0 prolene running sutures. Pressure water-seal test was performed to rule out air leak from the anastomosis with the two-lung inflation pressure of $30 \mathrm{cmH}_{2} \mathrm{O}$.

The total operation time was $210 \mathrm{~min}$, the high frequency jet ventilation time was $10 \mathrm{~min}$ without interruption, and the whole anastomosis procedure lasted $20 \mathrm{~min}$. The operation was successful, the oxygen saturation during the whole procedure was maintained at more than $98 \%$. Blood gas analysis showed a slightly elevated partial pressure of $\mathrm{CO}_{2}$ (Table 1). The patient was extubated in the operating room and monitored in postanesthesia care unit (PACU). Then she was cared in the intensive care unit for one night and was transferred to the general ward on the second day. No symptoms of respiratory distress were observed during the hospital stay. And the patient was discharged on the postoperative day 6 .

\section{Discussion}

The surgical procedures of right upper lobe sleeve and carinal resection and reconstruction is complicated. It remains a giant challenge for both the thoracic surgeons and the anesthesiologists. It requires precise airway management and coordination with the surgeons during airway resection and reconstruction. The satisfactory ventilation targets 
Table 1 Arterial blood gas analysis before, during and after the operation

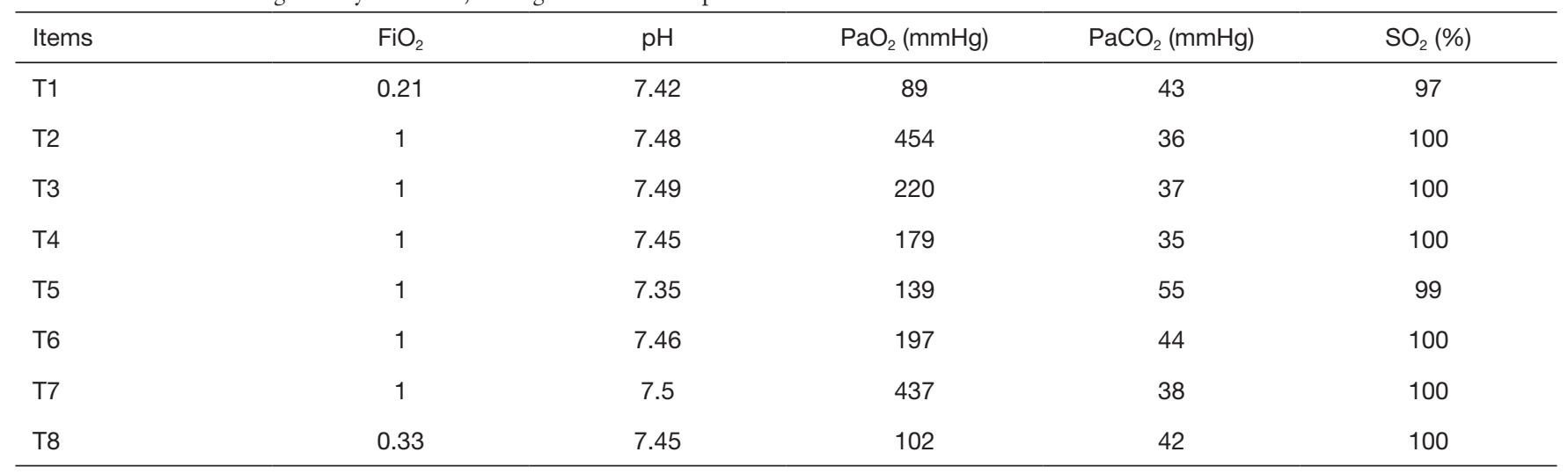

T1: 10 min before anesthesia induction breathing air. T2: 15 min after induction with two lung ventilation breathing $100 \%$ oxygen. T3: 15 min after one-lung ventilation initiation. T4: 5 min before high-frequency jet ventilation (HFJV) initiation. T5: 10 min after HFJV initiation (at the termination of HFJV). T6: $10 \mathrm{~min}$ after DLT one-lung ventilation. T7: 10 min after two-lung ventilation. T8: spontaneous breathing with $3 \mathrm{~L} / \mathrm{min} \mathrm{O}_{2}$ via a nasal catheter in the postanesthesia care unit (PACU).

include: one-lung ventilation with adequate gas exchange, right lung collapse to provide plenty surgical space, and no increase of the tension in suture during the anastomosis of the bronchi/trachea.

There are several methods of ventilation techniques for the tracheal and carinal resection surgery. Cross-field intubation was most commonly used, in which a sterile endotracheal tube was passed to the left main bronchus through an additional incision in the intercostal space to achieve one-lung ventilation (3). It guarantees adequate oxygen supply with the tidal volume, airway pressure and end-tidal $\mathrm{CO}_{2}$ partial pressure monitored in real-time. But it has the disadvantage of influencing the surgical operation, especially during anastomosis of the trachea, while uniportal VATS requires a clear exposure of the surgical field. Besides, the ventilation should be interrupted intermittently when the trachea is anastomosed using this technique. In the recent years, non-intubated spontaneous respiration anesthesia has been reported to be feasible for tracheal and carinal reconstructions with video-assisted thoracoscopic $(4,5)$. It provides a quite surgical view and reduces the intubation associated complications (6). The potential risks include hypoxemia, hypercapnia, uncontrolled coughing and soiling of the airway with blood and secretions. Considering the possible situations like tumour rupture and hemorrhage when dissecting the trachea, we decided that the general anesthesia with tracheal intubation was the optimal choice in this case.

We used a specially modified double-lumen endotracheal tube and left lung ventilation to facilitate the operation in this case, assisted with high frequency jet ventilation through a suction catheter to the left lung during the anastomosis of left main bronchus to the trachea. According to our clinical experiences, a size $35 \mathrm{Fr}$ left-sided DLT was recommended for an adult female with average body size in thoracic operations in general situations. In this case we chose a smaller size- $32 \mathrm{Fr}$ and reduced the balloon size of the tracheal cuff with the $3 \mathrm{M}$ film so it would not be in contact with the tracheal tumour and it would not increase the anastomosis tension of bronchus intermedius to trachea. Besides, the suction catheter could be easily inserted to the left or right lung from DLT and lessened the risks of aspiration of blood, sputum and tumour rupture.

During the anastomosis of left main bronchus to the trachea, high frequency jet ventilation was performed through the DLT. The small diameter of the endotracheal suction catheter connected to the HFJV permitted unimpaired visualization and adequate operating space in the surgical field. HFJV has the advantages of good gas exchange and reduced atelectasis due to its positive endexpiratory pressure (7). Using this technique, the second incision for the cross-field intubation was avoided, and the chance of contamination of the surgical field was greatly reduced. It should be noted that, arterial blood gas analysis should be closely monitored in case of hypoxemia and hypercapnia. If inadequate oxygenation occurs or the $\mathrm{PaCO}_{2}$ rises dramatically, the surgical procedure should be interrupted and the DLT remained in the trachea should be advanced to the left main bronchus to restart one-lung ventilation. Using this modified DLT, HFJV, conventional 
one-lung ventilation and two-lung ventilation could be easily switched. And the sterile endotracheal tube prepared in the surgical filed to implement cross-field ventilation was another emergency guarantee.

Along with advantages, our technique has its limitations. It is better be adopted when the longitudinal length of the carina being affected by the tumour is less than $3 \mathrm{~cm}$ to guarantee enough operating space and tumour free margins for the distance between the two cuffs is approximately $4 \mathrm{~cm}$. Also, it is more technically demanding for the operator as the duration of a single HFJV should be no more than $10 \mathrm{~min}$ in case of hypoxemia or hypercapnia. Although DLT can be advanced through the anastomosis to the left main bronchus to restore one-lung ventilation during this process, repetitive back and forth of the lumen tube should be avoided which may cause inflammation and edema of the margins.

In conclusion, this case shows that the modified doublelumen tube with the high frequency jet ventilation could provide satisfactory gas exchange and good surgical conditions. This kind of airway management may be a good option for the bronchial sleeve surgery and carinal resection and reconstructions under uniportal VATS in the future. More exploration and experience are needed to better define pros and cons of these techniques.

\section{Acknowledgments}

Funding: None.

\section{Footnote}

Conflicts of Interest: All authors have completed the ICMJE uniform disclosure form (available at http://dx.doi. org/10.21037/tcr.2019.12.90). The authors have no conflicts of interest to declare.

Ethical Statement: The authors are accountable for all aspects of the work in ensuring that questions related to the accuracy or integrity of any part of the work are appropriately investigated and resolved. All procedures performed in studies involving human participants were in accordance with the Declaration of Helsinki (as revised in 2013). Written informed consent was obtained from the patient for publication of this Case Report and any accompanying images. A copy of the written consent is available for review by the Editor-in-Chief of this journal.

Open Access Statement: This is an Open Access article distributed in accordance with the Creative Commons Attribution-NonCommercial-NoDerivs 4.0 International License (CC BY-NC-ND 4.0), which permits the noncommercial replication and distribution of the article with the strict proviso that no changes or edits are made and the original work is properly cited (including links to both the formal publication through the relevant DOI and the license). See: https://creativecommons.org/licenses/by-nc-nd/4.0/.

\section{References}

1. Guido-Guerrero W, Bolanos-Cubillo A, GonzalezRivas D. Single-port video-assisted thoracic surgery (VATS)-advanced procedures \& update. J Thorac Dis 2018;10:S1652-61.

2. He J, Wang W, Li J, et al. Video-assisted thoracoscopic surgery tracheal resection and carinal reconstruction for tracheal adenoid cystic carcinoma. J Thorac Dis 2016;8:198-203.

3. Weder W, Inci I. Carinal resection and sleeve pneumonectomy. J Thorac Dis 2016;8:S882-8.

4. Huang J, Qiu Y, Chen L, et al. Nonintubated Spontaneous Respiration Anesthesia for Tracheal Glomus Tumor. Ann Thorac Surg 2017;104:e161-3.

5. Peng G, Cui F, Ang KL, et al. Non-intubated combined with video-assisted thoracoscopic in carinal reconstruction. J Thorac Dis 2016;8:586-93.

6. Zhao ZR, Lau RWH, Ng CSH. Anaesthesiology for uniportal VATS: double lumen, single lumen and tubeless. J Vis Surg 2017;3:108.

7. Chin JH, Lee EH, Choi DK, et al. High frequency jet ventilation of one lung using a bronchial blocker of Univent during carinal resection. J Korean Med Sci 2010;25:1083-5.

Cite this article as: Dai L, Jiang L, Gu Y, Vannucci J, Lv X, Song J. Modified double lumen tube for a unique bronchial and carinal resection in a patient undergoing uniportal VATS for tumour: A case report. Transl Cancer Res 2020;9(3):2077-2081. doi: $10.21037 /$ tcr.2019.12.90 